\title{
A LORA-DRIVEN HOME SECURITY SYSTEM FOR A RESIDENTIAL COMMUNITY IN A RETIREMENT TOWNSHIP
}

\author{
Abubaker Sherif ${ }^{1}$, Suheib Sherif ${ }^{1}$, Chee Pun Ooi ${ }^{1}$, Wooi Haw Tan ${ }^{1 *}$ \\ ${ }^{1}$ Faculty of Engineering, Multimedia University, Persiaran Multimedia, 63100 Cyberjaya, Selangor, \\ Malaysia
}

(Received: November 2018 / Revised: January 2019 / Accepted: September 2019)

\begin{abstract}
The Internet of Things (IoT) has the capability to change our lives. In the near future, every device and almost every object will be linked together and connected to the internet. This paper summarizes the work done in developing a Long Range (LoRa) driven home security system for a residential community in a retirement township. Both hardware and software solutions were used to provide emergency alerts. A panic button was developed to generate alarms manually and embedded sensors were implemented to generate alarms automatically. LoRa wireless communication technology was used to connect all hardware devices in the network and to forward all these data to be stored in the server. A mobile application was developed to provide an alternative way of generating manual emergency alerts and to provide a communication channel in the form of a chat room for residents of the same community as well as the security guards. A web application was developed for residential area security management, where administrators can register new residential areas and assign security guards to these residential areas accordingly.
\end{abstract}

Keywords: Home security; IoT; LoRa; Residential community; Smart cities

\section{INTRODUCTION}

The Internet of Things (IoT) has the capability to alter our way of life. In the near future, every device we own and almost every object around us will be linked together and connected to the internet. The IoT will join us in ways we currently cannot even fathom (Berawi, 2018). By 2050, approximately $66 \%$ of the world's population will be living in urban areas, which offer basic resources such as good food, high security, quality health care, and sufficient energy. In this new world, providing overall economic and environmental sustainability will be our biggest challenge, and efficiency in the allocation of these basic resources will need to be enhanced (ISO, 2018).

The demographic change in our societies is one of the major challenges that we are now facing, according to the World Health Organization (WHO, 2002). The population is aging worldwide, and this will have a great impact on future generations, especially on the economy and on productivity. Recent research has shown that the percentage of people who are 65 years old and above is increasing and will reach $29.5 \%$ in 2060, compared to $17.4 \%$ in 2010 (European Commission, 2011). The safety of the older population is a concern because many older people spend most of their time at home and alone, leaving them more vulnerable to property thefts and other crimes. The probability of theft in residential neighbourhoods can be reduced if a proper

*Corresponding author's email: twhaw@mmu.edu.my, Tel. +603-83125447, Fax. +603-83183029

Permalink/DOI: https://dx.doi.org/10.14716/ijtech.v10i7.3257 
emergency alarm system is implemented.

The increasing elderly population presents numerous challenges for smart cities. Handling security quality and its costs is one of the most important considerations in the design and implementation of smart cities, but community councils should consider other issues as well. Security for senior citizens and elderly people can be one of the most significant challenges for smart residential areas, and the increasing number of retired persons will force smart cities' community councils to assist this demographic group and work for its benefit. These councils should carefully plan the assistance they provide to these retired people, so that they can live the rest of their lives in safety and comfort. Advanced security systems for retirement residential homes are essential. This project is designed to maximize the efficiency of security systems and make those systems effective and easy to use by retired people. Retired people should be able to live in peaceful homes that offer them a safe environment and protection from the dangers of the outside world. Modern technologies, such as the expansion of the IoT, are driving the development of smart cities and industries, especially in the areas of cost reduction and quality improvement (Suwartha, 2017).

Retired citizens deserve to stay at home in a peaceful environment that offers them safe lives and protection from strangers. This project proposes a security system in an intelligent residential area that ensures a safe and convenient living environment for retired people. Long Range (LoRa) wireless technology is used in this security system. Our security system is a simple and interactive management system that is designed to allow security guards and the community council to monitor the status of each family in the residential area. A mobile application was developed for use by family members in case of emergency. We also considered the issue of sustainability, because investors and other financial stakeholders need an efficient financial plan in order to provide viable solutions to problems. Two modes of emergency calls were enabled in this security system, one a human-generated alarm and the other an automated sensor alarm. This project made efficient use of LoRa wireless network, which sends short messages for long ranges using low power. However, it is not meant to handle high data rates or send large amounts of data in each message

\section{LITERATURE REVIEW}

In order to develop an efficient security system, we reviewed some previous solutions from different perspectives, such as hardware devices and the technology used in data communication.

\subsection{Related Solutions}

Plentiful research has been conducted on home security systems, which have become important to modern life (especially for people who retired after decades in the workforce and chose to live alone in retirement townships). Many proposed systems allow community residents to call for assistance, but not all of them provide emergency alerts to nearby authorities, offering only local alarms within the homes (Sharma et al., 2014; Kulsiriruangyos et al., 2016). Some solutions feature nothing more than a mobile application to send an emergency call, while others use automated sensors to send an automatic emergency alarm without any human interaction (Sharma \& Gogoi, 2013; Hong et al., 2016). Several technologies have been used to address these problems, such as Wi-Fi communications, transmitting over radio frequencies, the Global System for Mobile Communications, smartphone applications, the Global Positioning System, and the Public Switched Telephone Network (Sharma \& Gogoi, 2013; Marek \& Peter, 2014; Choudhury et al., 2015). Each use of these technologies has its advantages and disadvantages, depending on how they are implemented by the developer. 


\subsection{Related Products}

Many companies and governments are currently investing a lot of money to improve security in residential areas and assist as many people as possible in getting the help they need during emergency situations (Careline365, 2018; MERS, 2018). Different methods have been used to solve this problem. Some products include the software method only by using a smartphone application, while others use hardware as well (Protect Your Home, 2018; Occly Security, 2018). Several other technologies have been used to address the issue of security in residential areas. Efficient use of technology is vital to limit the cost of any solution and to decrease the complexity of the system.

\section{DETAILS OF THE DESIGN}

After examining previous research solutions and products on the market and highlighting their strengths and weaknesses, we have designed an optimal solution to overcome the weaknesses of other schemes and implement a stable, secure, and reliable security system.

\subsection{System Architecture}

The overall system architecture is shown in Figure 1. Home owners must first register their houses in the system by providing personal information, such as the name of the residential area, unit number, contact number, full name, and e-mail.

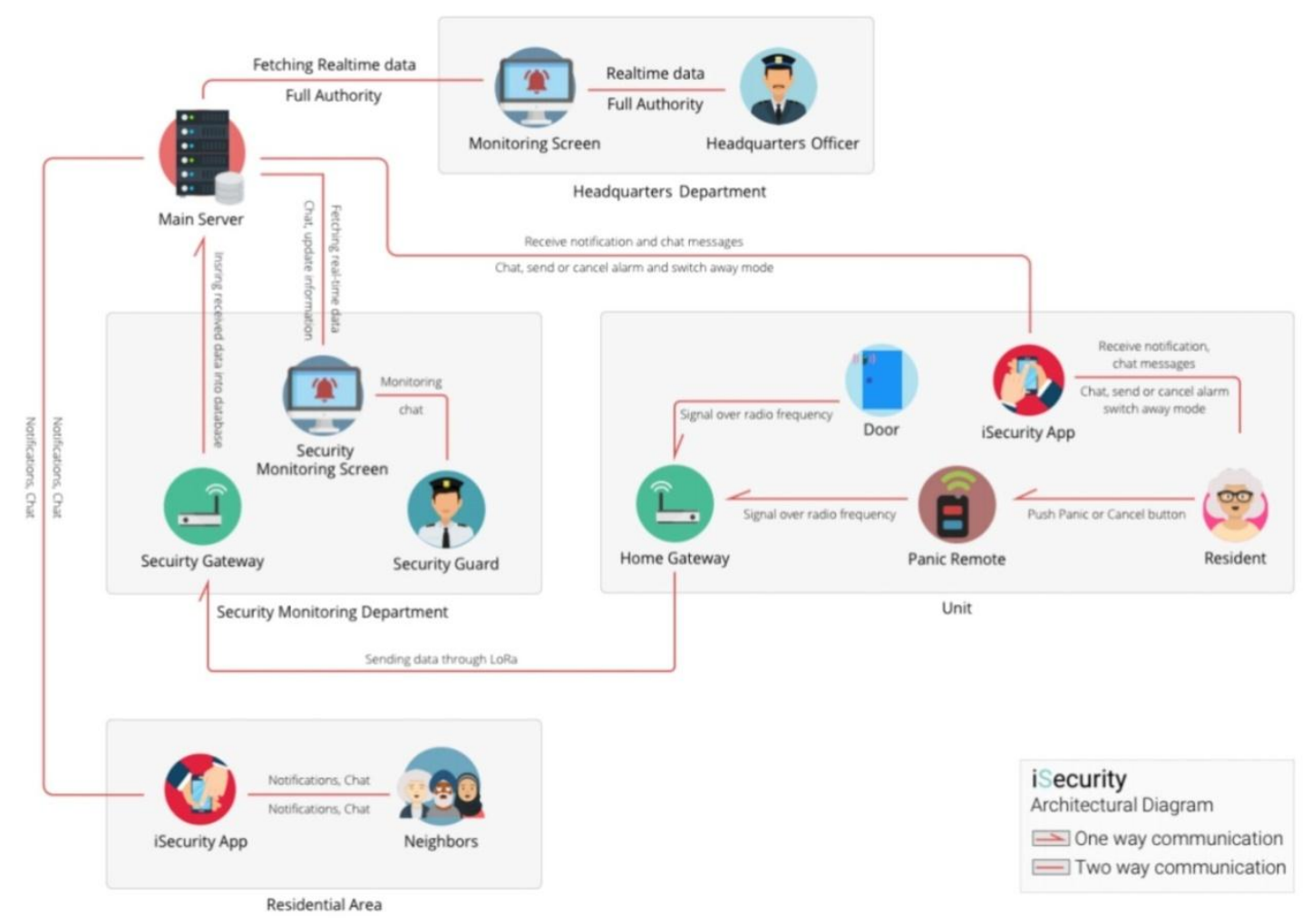

Figure 1 System architecture

The user must scan a barcode in order to download our mobile application, which is called iSecurity. The headquarters security officer will then give the user a password to sign in to the smartphone application in order to use the service. In case of emergency, the user has two ways to send an alarm to the security guards. The first is via the panic button device, which will send a signal to the home gateway that will be forwarded to the security office gateway. Once the message is received by the security office gateway, the message will be published to the database 
in the server and the web application viewed by the security guard will show and play an alarm notification. The webpage displayed in the security office will show the unit number, home owner name, and location of the unit, allowing help to reach the victim. The device also offers a cancel button, which can be used if the panic button is pressed accidentally.

The user also has a second way to send an emergency alarm with a smartphone. The application installed in the user's phone has the ability to send an alarm to the security guard using a panic button in the application. The message will be sent to the database immediately to notify the security guards in the security office of the situation, the unit number, the home owner name, and the location of the unit sending the alarm. The user always has an opportunity to cancel any alarm sent mistakenly by pressing the cancel button in the mobile application. A third way to send an alarm message to the security guards is by using an automated sensor attached to the door of the house. The sensor will send an alarm signal to the home gateway, which will forward the message to the security office gateway.

Once the message is received by the security office gateway, the message will be pushed to the database in the server and the web application viewed by the security guard will show and play an alarm notification. This mode should be activated using the smartphone application only when the user is away from home or sleeping and not expecting visitors; at other times, the user can deactivate the away mode so any activity detected by the sensor will be ignored. The user can also communicate with the security guards of the residential area as well as their neighbours by using the iSecurity application to inquire about any action happening in the area. Whenever an alarm message is received by the security guard monitor, everyone living in that same residential area will receive a notification on the mobile application with the number of the unit seeking help. The user may either ignore the notification or redirect it to the chat room in the iSecurity application to learn about any causes for concern. Using the smartphone application, the user can switch between two modes: home and away. Activating home mode will ignore alarms sent from the embedded sensors and only accept the alarms from the manual panic button. Conversely, away mode allows users to receive alarms from the embedded sensors.

\subsection{System Design}

This system is made using many technologies and techniques to guarantee efficient and reliable communication between different elements of the security system. The main technology used in this proposed solution is LoRa (Blenn \& Kuipers, 2017). Several technologies and software were used to obtain high performance, efficiency, reliability, and low cost, with a simple user interface for both home owners and the security guards who monitor this system. These technologies are Remote Dictionary Server (Redis), Advanced Encryption Standard (AES), Model-ViewController (MVC), and background workers (Das, 2015; Lin et al., 2016).

\subsection{Hardware Implementation}

\subsubsection{Security gateway}

This part of the hardware is based on an Arduino Nano microcontroller with an ATmega328 processor. This microcontroller board is connected with multiple modules, an in Air9b wireless module for LoRa communications, and two radio frequency receivers (one to receive a signal from the panic button device and the second to receive a signal from the door sensor), as shown in Figure 2.

The panic button receiver accepts two types of signal: alarm and cancel. The board is programmed to wait for the radio frequency receivers to be triggered. Once a receiver is triggered, the processor will pass a string type of encrypted data to the wireless module containing the type of message and the user id dedicated to the house sending the signal. 


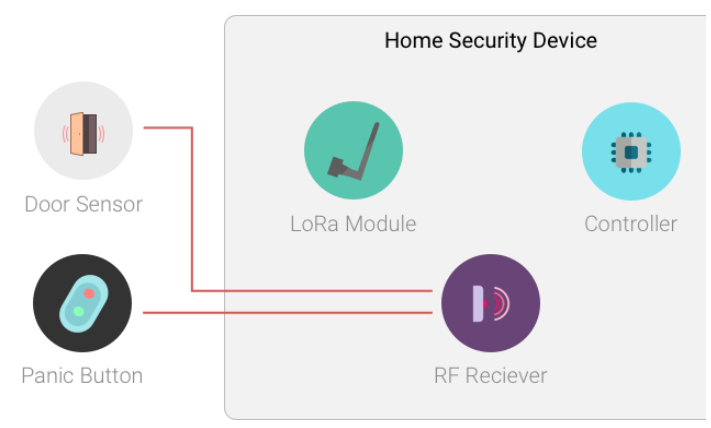

Figure 2 Home gateway hardware design

\section{- Panic Button Device}

This part of the hardware consists of a radio frequency transmitter connected to two push buttons, one to send an alarm signal and one to send a cancel signal, as shown in Figure 2. When the user presses one of the buttons, the RF module will transmit a signal to the receiver side.

\section{- VibrationSensor Device}

This part of the hardware is based on an Arduino mini pro microcontroller with an ATmega328 processor. This microcontroller board is connected to a radio frequency transmitter and a vibration sensor. The board is programmed to wait for the sensor to detect a vibration. The sensor sends a value of vibration to the microcontroller, and once the sensor exceeds a threshold value, the microcontroller will trigger the RF transmitter to send a signal to the receiver side of the home gateway.

\subsubsection{Central gateway}

This part of the system is based on an Arduino Nano microcontroller with an Atmega328 processor. This microcontroller board is connected to an in Air9b wireless module for long range wireless communication protocol and is also connected serially to a Raspberry Pi Zero W using a USB cable. The security gateway components are shown in Figure 3.

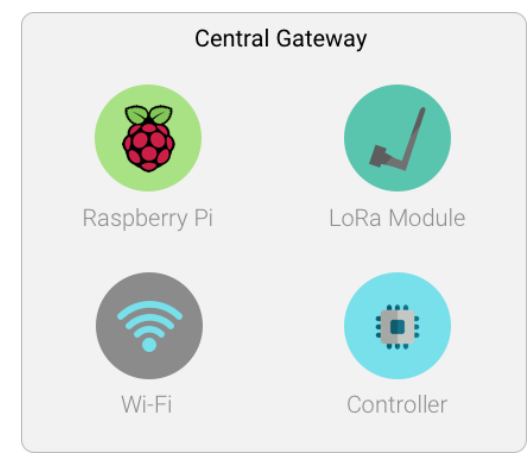

Figure 3 Central Gateway

The data is received through the LoRa module and decrypted by the processor of the Arduino board in order to be transferred serially by a USB cable to the raspberry pi. The processor of the raspberry pi formats the received message in a JSON file format in order to push the JSON data in a Redis queue. This process is repeated for each alarm signal received by the LoRa module.

\subsection{Software Implementation}

This part of the system handles the processing of the data (where the data are categorized and stored) as well as the generating of alarm notifications to both web and mobile applications. The server is responsible for sequencing the data for transfer, so that it becomes easier for front-end applications to deal with stored data. 


\subsubsection{Web application}

This part of the system is implemented to assist administrators in registering new residential areas and to monitor real-time alarms generated by residents in the form of a map view displaying the location of the unit seeking help. It also allows security guards in each security area to register new residents and observe real-time resident alarm notifications in the same map-view format. This web application offers security guards in each residential area a real-time chat room to interact with residents in case of emergency or in case any resident is seeking help, and this creates a form of community for the entire residential area.

\subsubsection{Mobile application}

This part of the system has been developed to assist resident users by generating an alarm notification sent to the monitoring centre and neighbours in the same residential area and by providing the ability to cancel any notification in the event of a false alarm. It also offers a realtime chat room to help residents communicate with security guards in case of emergency or to ask about any situation of concern.

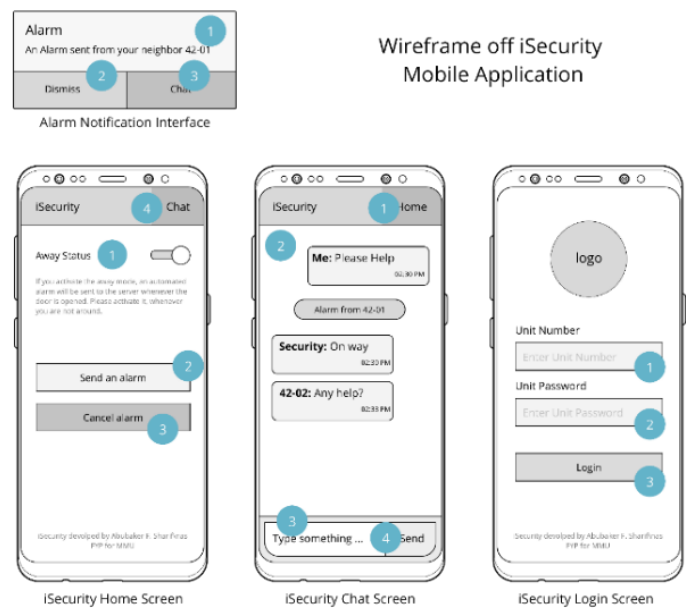

Figure 4 Mobile application

Figure 4 illustrates the main activities and functionalities of the developed mobile application. The first screen shows the main activity of the application, where the user interacts with its four main functionalities. The first function is the Send Alarm button; clicking that button creates an emergency event that is queued in the system to call for help.

The second functionality is the Cancel Alarm button, which sends a notification to the system to ignore any initiated alarm for this user. The third functionality is Away Status, a toggle type of input where the user has the option to either turn on the sensor alarm signals or ignore them from the system as described earlier. The fourth function is the button to enter the chat room (at the top). The chat room includes all residents from the same area as well as the security guards.

\section{SYSTEM IMPLEMENTATION}

The designed system can be practically implemented in a retirement residential area as shown in Figure 5, where the complex is equipped with an iSecurity gateway and each house is assigned an iSecurity home device with panic buttons and door sensors. Residents can use the mobile application to contact other residents in case of emergency or send alarm signals to the security guards. Through the mobile application, all residents receive notifications in case of emergency along with information on which house is calling for help, allowing residents to assist each other. 


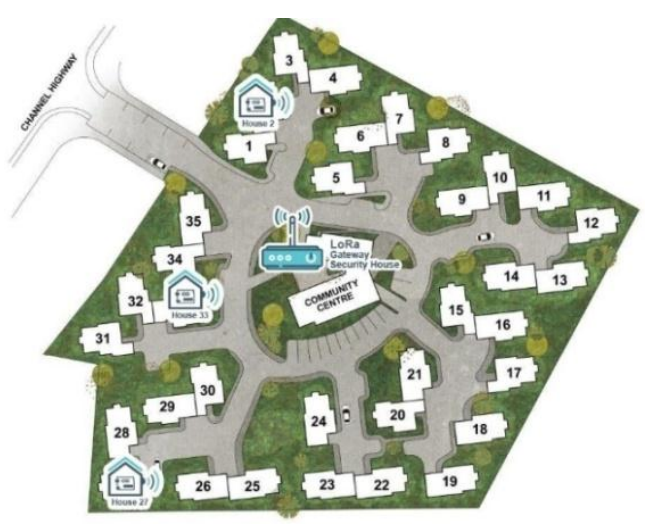

Figure 5 System Implementation

In order to evaluate the designed system, a practical prototype was developed by integrating all hardware and software components of the system. The web application proposed is run on a LAMP server bundle furnished by Digital Ocean cloud provider. A LAMP server is basically a Linux server running Apache for web applications, MySQL for structured database storage, and PHP for web applications and background workers. The development of the complete web application was carried out using Laravel MVC framework to manage all resources.

\section{RESULTS AND DISCUSSION}

Both hardware and software tests were conducted in order to verify the system functionalities as well as testing the reliability and throughput. Further tests were conducted to examine the LoRa network, especially its range and data rate capabilities. Hardware tests included range, latency, power consumption, and scalability. For the software testing, a full functionality test was done to verify that each task was carried out successfully and to show that the overall system is functioning as designed and serving the correct purpose.

\subsection{Server Heavy Load Test}

A heavy load test was done on the server side to test how much alarm traffic the system could handle in a given time.
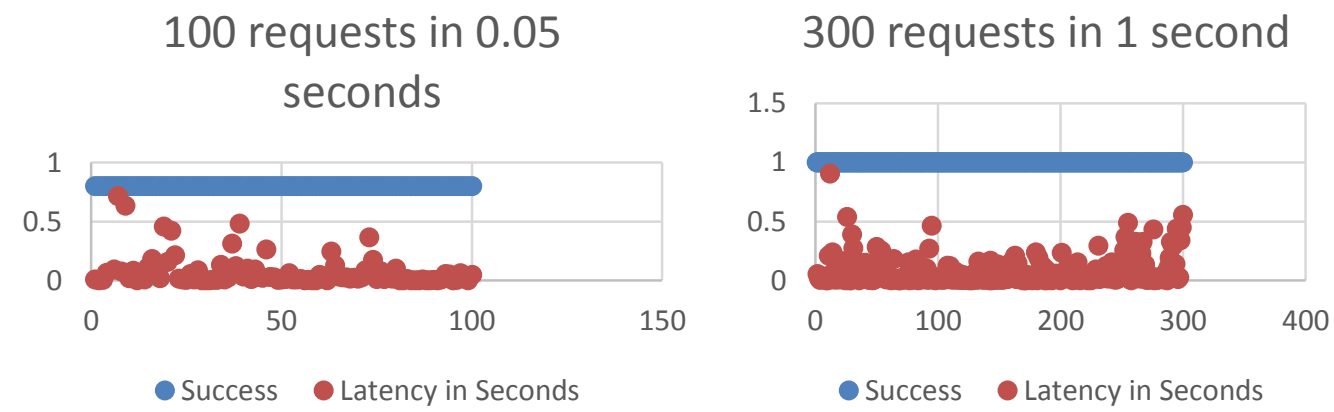

Figure 6 Heavy Load Server Tests part 1

The results shown in Figure 6 depict strong performance in handling up to 100 alarm requests in a short period of time, almost simultaneously and an even higher load of 300 successful server requests in one second. Response latency showed great performance as well, with maximum response delay just below one second for only a few requests. 


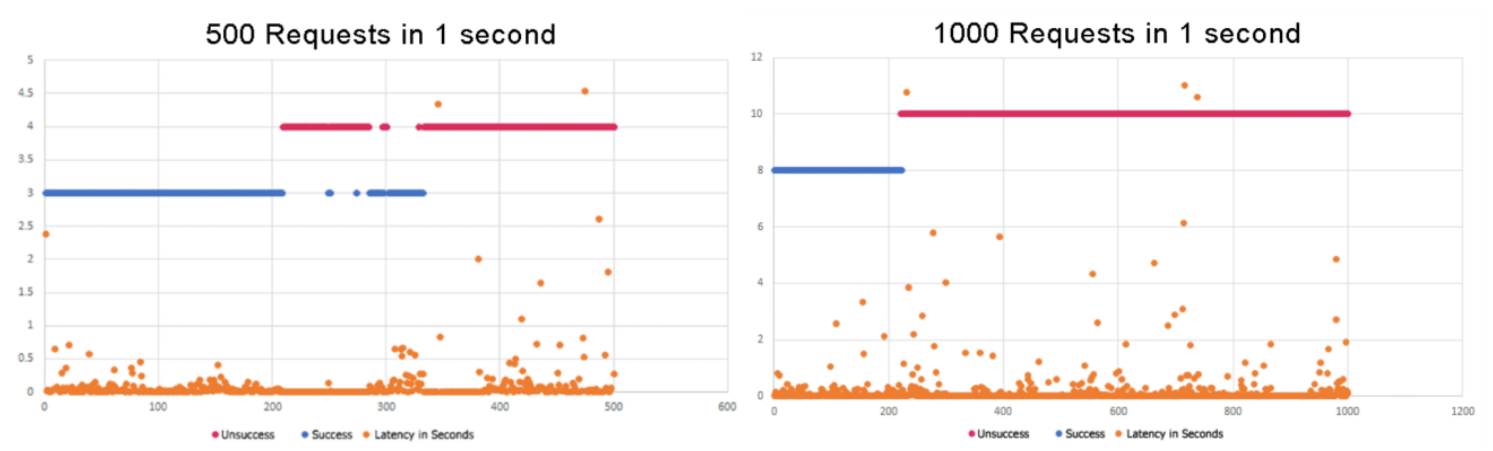

Figure 7 Heavy Load Server Tests part 2

In order to further examine system performance, a more condensed test was done with 500 requests in one second for the first part and 1,000 requests in one second for the second part. The results shown in Figure 7 illustrate a similar pattern with successful response for the first 200 requests. After that, the system performance degraded; the server could not handle the remaining requests with occasional successful requests in between. It was also noticed that system response latency increased significantly when the system started to experience unsuccessful requests, which reached up to 11 seconds for a single response.

\subsection{Overall Latency Test}

A manual latency test was conducted to examine the overall system latency in generating alarms from both the mobile app and system hardware.

Table 1(a) Mobile latency tests

\begin{tabular}{cc}
\hline $\begin{array}{c}\text { Attempts to } \\
\text { send request }\end{array}$ & $\begin{array}{c}\text { Time in Seconds } \\
(\mathrm{App})\end{array}$ \\
\hline 1 & 1.48 \\
2 & 1.39 \\
3 & 1.60 \\
4 & 1.73 \\
5 & 1.50 \\
6 & 1.55 \\
7 & 1.93 \\
8 & 1.88 \\
9 & 1.80 \\
\hline
\end{tabular}

Table 1(b) Hardware latency test

\begin{tabular}{cc}
\hline $\begin{array}{c}\text { Attempts to } \\
\text { send request }\end{array}$ & $\begin{array}{c}\text { Time in Seconds } \\
\text { (App) }\end{array}$ \\
\hline 1 & 1.9 \\
2 & 1.8 \\
3 & 1.9 \\
4 & 1.7 \\
5 & 2.0 \\
6 & 1.8 \\
7 & 1.9 \\
8 & 1.9 \\
9 & 2.1 \\
\hline
\end{tabular}

Table 1a shows the results of the mobile app latency test, where multiple alarms were sent and the time between generating the alarm and receiving the alarm on the dashboard was measured. It demonstrated good performance, with response time between 1.39 and 1.93 seconds. The hardware latency test in Table 1b showed similar behaviour, with latency between 1.7 and 2.1 seconds, which is considered good in such an application.

The web application homepage reproduced in Figure 8 displays a map that shows the location of any alarm notification received to the website as well as a real-time chat room to interact with residents in the area. Figure 8 also shows the home screen activity of the iSecurity application, which contains the panic button, cancel alarm button, and switch button to activate and deactivate the away mode. The second screen is the chat screen activity, where the user can interact with neighbours and security guards in case of emergency. 

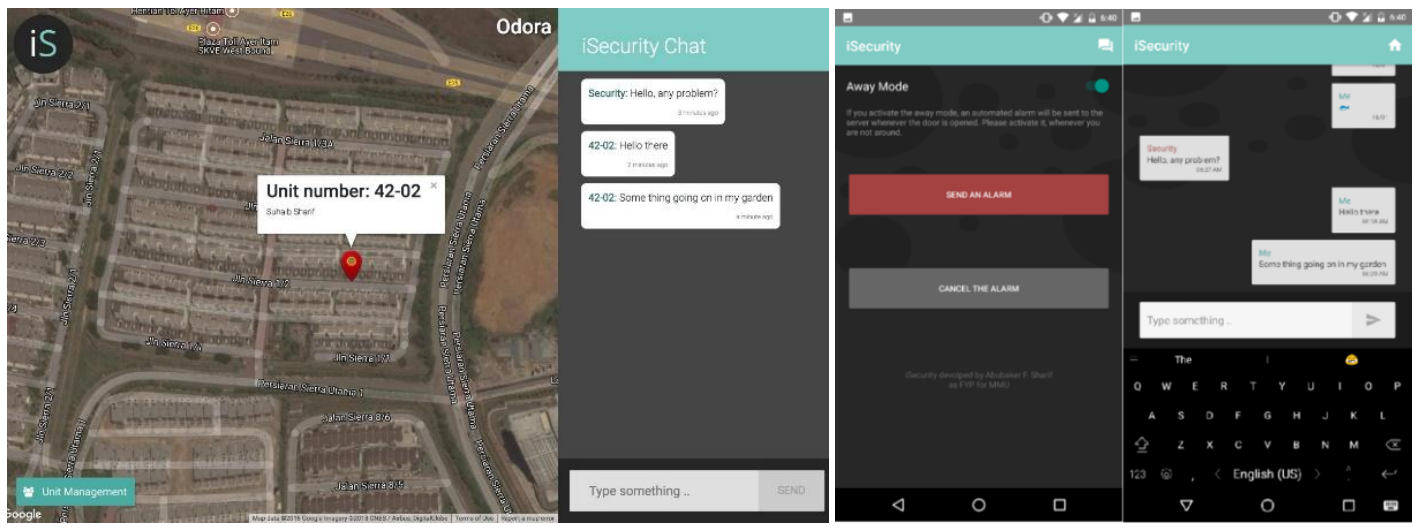

Figure $8 \mathrm{Web}$ application and residents mobile application

The main contributors to the overall http request processing latency are the server RAM, the CPU processing speed and number of cores, and the drive speed. The operating system and server configuration also play dominant roles in the overall delay. Server network bandwidth also has a direct effect on server performance. Other factors, such as Firewalls, DNS, and other connection configurations, affect the system response. The app relies on constant, real-time exchange of information between the server and the user in different regions, and this communication is powered by internet networks. Because network performance varies based on network conditions, so does app performance. Other factors, such as apps running in the background, battery time while using the app, and other mobile configurations, also affect system response time. The end device hardware performance relies on many variables, but the main one is the LoRa network coverage, where system performance varies based on the distance from the gateway. Another factor is the specifications of controllers running the device, such as flash memory and micro controller clock speed.

In short, for the designed system, these results show satisfactory outcomes and the latency is low enough for alarm applications.

\section{CONCLUSION}

Research was conducted to identify current and previous efforts in this area and to learn the current trends and technologies that are driving modern security solutions. An overall system architecture was then designed in order to visualize the complete distributed system. Afterwards, the detailed system design was highlighted, and each part of the system was explained along with its purpose and how it was connected to the other parts. After developing the designed prototype, a complete system test was performed, and the results have shown that the system is suitable for implementation in a residential area. The system is expected to perform the designed task completely.

\section{REFERENCES}

Berawi, M.A., 2018. The Fourth Industrial Revolution: Managing Technology Development for Competitiveness. International Journal of Technology, Volume 9(1), pp. 1-4

Blenn, N., Kuipers, F., 2017. LoRaWAN in the Wild: Measurements from the Things Network. Available Online at https://arxiv.org/abs/1706.03086, Accessed on February 20, 2018

Careline365, 2018. Available Online at https://www.careline.co.uk., Accessed on February 20, 2018

Choudhury, B., Choudhury, T.S., Pramanik, A., Arif, W., Mehedi, J., 2015. Design and Implementation of an SMS Based Home Security System. In: IEEE International Conference on Electrical, Computer and Communication Technologies (ICECCT) 2015, pp. 1-7 
Das, V., 2015. Learning Redis. Packt Publishing Ltd., Chicago, Illinois, USA.

European Commission, 2011.The 2012 Ageing Report: Underlying Assumptions and Projection Methodologies. Office for Official Publications of the European Communities, Brussels, Belgium

Hong, X., Yang, C., Rong, C., 2016. Smart Home Security Monitor System. In: IEEE $15^{\text {th }}$ International Symposium on Parallel and Distributed Computing (ISPDC) 2016, pp. 247 251

ISO, 2018. New Global Platform to Help Cities Become Sustainable and Smart. Available Online at https://www.iso.org/news/2016/01/Ref2042.html, Accessed on February 20, 2018

Kulsiriruangyos, J., Rattanawutikul, V., Sangsartra, P., Wongsawang, D., 2016. Home Security System for Alone Elderly People. In: IEEE Fifth ICT International Student Project Conference (ICT-ISPC) 2016, pp. 65-68

Lin, J., Lin, L.C., Huang, S., 2016. Migrating Web Applications to Clouds with Cloud-based MVC framework. In: IEEE International Symposium on Computer, Consumer and Control (IS3C) 2016, pp. 1039-1042

Malaysia Emergency Response Services MERS 999, 2018. Available Online at http://www.999.gov.my, Accessed on February 20, 2018

Marek, G., Peter, S., 2014. Design the Robot as Security System in the Home. Procedia Engineering, Volume 96, pp. 126-130

Occly Security, Security \& Civilian Bodycam, 2018. Available Online at https://www.occlysecurity.com, Accessed on February 20, 2018

Protect Your Home, ADT Home Security and Alarm SYSTEMS, 2018. Available Online at https://www.protectyourhome.com, Accessed on February 20, 2018

Sharma, R.K., Gogoi, D., 2013. Android Based Emergency Alert Button. Available Online at https://arxiv.org/abs/1311.5133. Accessed on February 20, 2018

Sharma, R.K., Mohammad, A., Kalita, H., Kalita, D., 2014. Android Interface-based GSM Home Security System. In: IEEE International Conference onIssues and Challenges in Intelligent Computing Techniques (ICICT) 2014, pp. 196-201

Suwartha, N., Ardiyansyah, Berawi, M.A., Surjandari, I., Zagloel, T.Y.M., Atmodiwirjo, P., Yatmo, Y.A., 2017. Science, Technology and Innovation for Sustainable World. International Journal of Technology, Volume 8(6), pp. 979-982

WHO, 2002. Active Aging, A Policy Framework. World Health Organization, Geneva Switzerland 